Reprinted from Journal of the Franklin Instutute, Vol. 279, No. 2, February, 1965 Printed in U. S. A.

\title{
Internal Instability of Anisotropic Viscous and Viscoelastic Media Under Initial Stress
}

by M. A. BIOT

Shell Development Company

New York, N. Y.

ABSTRACT: The internal instability of anisotropic viscoelastic media under initial stress is investigated in analogy with the phenomenon of internal buckling in elasticity by applying the author's principle of viscoelastic correspondence. The spreading of a disturbance in a medium of infinite extent is analyzed. A numerical solution is derived which describes the time history of an initial perturbation in a purely viscous anisotropic medium in compression and confined between parallel boundaries. It is shown that the system acts as a high-pass filter on perturbations of various wavelengths. In the range of significant instability the solution obtained by viscoelastic correspondence from the theory of elasticity is found to be applicable as an approximation to a viscous anisotropic fluid initially in a state of flow. An exact and independent treatment of the latter case is derived for comparison.

\section{Introduction}

In a previous paper (1) we have shown that an elastic medium confined by rigid boundaries and under initial stress may exhibit an instability referred to as internal buckling. It was established that there are two types of internal buckling which we have proposed calling internal instability of the first and second kind (2). The former may occur only in a material of intrinsic anisotropy. 
A similar internal instability is to be expected in viscoelastic media. In particular we are concerned here with a phenomenon analogous to the internal instability of the first kind. Hence we consider a viscoelastic medium of orthotropic anisotropy under an initial compression acting along one of the axes of symmetry.

The problem is analyzed by applying the principle of viscoelastic correspondence first established by the author in 1954. By this principle the elastic solution is applicable to viscoelasticity. This correspondence is rigorously valid when the undisturbed medium is at rest under the initial stress (2).

In the next section the elastic solution is briefly recalled. The viscoelastic medium of infinite extent is analyzed in section 3 showing the existence of a fan-like region of characteristic directions wherein disturbances spread out gradually.

A different type of phenomenon is examined in section 4 by considering a medium confined between two rigid boundaries parallel to the direction of the compression. An initial disturbance of simple distribution is assumed and the redistribution and amplification is evaluated as a function of time. The analysis has been carried out numerically for the particular case of a medium which is purely viscous for incremental deformations and may be considered as representing a certain type of plasticity. If the medium is at rest when undisturbed under the initial stress, the principle of viscoelastic correspondence applies rigorously and the analysis is exact for this case. However, the result also provides a good approximation where initial flow is present. It may be particularly applied to an anisotropic viscous fluid and the analysis is carried in the context of this approximation. The validity of the approximation is confirmed by an exact analysis of the anisotropic fluid in section 5 .

The problem of internal instability of a viscous medium between rigid boundaries brings out a physical feature of particular interest. Because of the confinement, disturbances of large wavelengths are restrained and the amplification will be restricted to the shorter wavelength components. The system therefore behaves as a filter. Hence in a qualitative way we may speak of a cutoff wavelength. The gradual appearance of an oscillation in the unstable disturbance is typical of such filtering and shows up in the numerical analysis in section 4 .

As an example of anisotropy we also consider a thinly laminated medium constituted by an alternation of hard and soft layers. However, to treat this material as a continuous medium is an approximation valid only if the layers are very thin compared to the wavelength of the disturbance. Further elaboration on this problem will be found in (2) and (3).

\section{General Equations}

Consider an homogeneous incompressible medium of orthotropic symmetry. The deformation is restricted to one of plain strain defined by two displacement components $u$ and $v$ in the $x, y$ plane. The strain is defined by 


$$
\begin{aligned}
& e_{x x}=\frac{\partial u}{\partial x} \quad e_{y v}=\frac{\partial v}{\partial y} \\
& e_{x y}=\frac{1}{2}\left(\frac{\partial v}{\partial x}+\frac{\partial u}{\partial y}\right)
\end{aligned}
$$

and the local rotation by

$$
\omega=\frac{1}{2}\left(\frac{\partial v}{\partial x}-\frac{\partial u}{\partial y}\right) .
$$

Incompressibility is expressed by the condition

$$
e_{x x}+e_{y y}=0
$$

The $x$ and $y$ directions are assumed to be axes of symmetry for the physical properties. The medium is under initial stress with principal stresses along $x$ and $y$ equal to $S_{11}$ and $S_{22}$. The deformation Eq. 1 generates stress increments $s_{11}, s_{22}$ and $s_{12}$ referred to local coordinate axes which are rotated with the medium through the angle $\omega$. The equilibrium conditions for the incremental stresses are $(1,2)$

with

$$
\begin{aligned}
& \frac{\partial s_{11}}{\partial x}+\frac{\partial s_{12}}{\partial y}-P \frac{\partial \omega}{\partial y}=0 \\
& \frac{\partial s_{12}}{\partial x}+\frac{\partial s_{22}}{\partial y}-P \frac{\partial \omega}{\partial x}=0
\end{aligned}
$$

$$
P=S_{22}-S_{11}
$$

If $S_{22}=0$, a positive value of $P$ represents a compression in the $x$ direction.

For an elastic medium we have shown $(1,2)$ that the stress-strain relations are

$$
\begin{aligned}
s_{11}-s & =2 N e_{x x} \\
s_{22}-s & =2 N e_{y y} \\
s_{12} & =2 Q e_{x y} .
\end{aligned}
$$

In previous work (1) we have also considered two other elastic coefficients defined as

$$
\begin{aligned}
M & =N+\frac{1}{4} P \\
L & =Q+\frac{1}{2} P .
\end{aligned}
$$

The physical significance of $M$ is obtained by considering an initial compression $P$ along $x$ and an incremental tensile force $t_{11}$ in the same direction per unit initial area. Then

$$
\iota_{11}=4 M e_{x x}
$$

The other coefficient $L$ which we have called the slide modulus, is derived by considering tangential forces $\Delta_{x y}$ applied on the free faces of a strip of material 
cut along $x$. The shearing deformation is given by

$$
\Delta_{x y}=2 L e_{x y} .
$$

For a viscoelastic medium the stress-strain relations are formally identical to those of the elastic medium and are obtained by replacing the elastic coefficients by operators. The general validity of this correspondence principle for isotropic and anisotropic media was derived by the author in 1954 in the context of thermodynamics. It was also shown that suitable elastic coefficients must be chosen in order to satisfy thermodynamic principles $(2,4)$. In this case suitable coefficients are $M$ and $L$. The corresponding operators are written

$$
\begin{aligned}
& \bar{M}=\int_{0}^{\infty} \frac{p}{p+r} M(r) d r+M+p M^{\prime} \\
& \bar{L}=\int_{0}^{\infty} \frac{p}{p+r} L(r) d r+L+p L^{\prime},
\end{aligned}
$$

where all quantities $M(r), M, M^{\prime}$ and $L(r), L, L^{\prime}$ are non-negative. The symbol $p$ denotes the differential operator

$$
p=\frac{d}{d t},
$$

where $t$ is the time variable. The significance of these operational expressions is illustrated by the simple example

$$
\frac{p}{p+r} e_{x x}=\int_{0}^{t} e^{-r(t-\tau)} d e_{x x}(\tau)
$$

which represents an exponential heredity function. We may also put $p$ equal to a real or complex quantity. In this case the operators become algebraic quantities and correspond to solutions proportional to the factor $\exp (p t)$.

In the discussion of internal instability for the elastic case (1), we investigated solutions represented by a displacement field of the type

$$
\begin{aligned}
& u=C \frac{\pi}{l h} \sin l x \sin \frac{\pi y}{h} \\
& v=C \cos l x \cos \frac{\pi y}{h} .
\end{aligned}
$$

This identical solution is given in Eq. 7.3 of (1) except for the trivial difference resulting from a translation along $x$. The displacement field Eq. 13 contains an arbitrary amplitude factor $C$, and satisfies condition Eq. 3 of incompressibility. This was found $(1,2)$ to satisfy Eqs. 1, 2, 4 and 6 provided

$$
P=L \xi^{4}+2(2 M-L) \xi^{2}+L .
$$

The variable $\xi$ is

$$
\xi=\frac{\pi}{l h}=\frac{\mathscr{L}}{2 h} .
$$


The field Eq. 13 may be looked upon as a mode of internal buckling of wavelength $\mathcal{L}$ along $x$ and wavelength $2 h$ along $y$. It may represent the behavior of a medium of infinite extent along characteristic directions as discussed in section 3 or the case of a confined medium between two frictionless rigid walls separated by a distance $h$ as discussed in section 4 . In both cases the medium is under an initial compression $P$ in the $x$ direction.

For a viscoelastic medium the same solution is valid except that the constant $C$ must be multiplied by the exponential factor $\exp (p t)$. By viscoelastic correspondence the characteristic Eq. 14 becomes

$$
P=\bar{L} \xi^{4}+2(2 \bar{M}-\bar{L}) \xi^{2}+\vec{L} .
$$

This is a relation between the wavelength $\mathcal{L}$ and the parameter $p$. Hence it determines the exponential rate of growth of the displacement field $u, v$ for any given wavelength.

An anisotropic continuous medium may be considered to approximate the properties of a material constituted by thin layers of alternating rigidity. The limitations of this approximation were discussed in $(2,3)$. The approximation is valid if the layers are sufficiently thin relative to the wavelength of the deformation field. The elastic coefficients for a thinly layered elastic medium were derived in (1). The result is immediately extended to viscoelastic media by the correspondence principle. Assume the layers are made of two different materials, one characterized by the operators $\bar{M}_{1} \bar{L}_{1}$ and occupying a fraction $\alpha_{1}$ of the total thickness, the other characterized by the operators $\bar{M}_{2} \bar{L}_{2}$ and occupying a fraction $\alpha_{2}$ of the total thickness. By definition we have the relation

$$
\alpha_{1}+\alpha_{2}=1
$$

The operators of the composite medium are then

$$
\begin{aligned}
\bar{M} & =\alpha_{1} \bar{M}_{1}+\alpha_{2} \bar{M}_{2} \\
\bar{L} & =\frac{1}{\frac{\alpha_{1}}{\bar{L}_{1}}+\frac{\alpha_{2}}{\bar{L}_{2}}} .
\end{aligned}
$$

Specific examples of composite media will be discussed. The initial compressive stresses in a direction parallel to the layers are denoted by $P_{1}$ and $P_{2}$ respectively in each material. Hence,

$$
P=\alpha_{1} P_{1}+\alpha_{2} P_{2}
$$

is the average initial stress in the composite medium.

\section{Internal Instability of a Viscoelastic Anisotropic Medium}

We shall discuss the instability for a particular type of viscoelastic medium. Let the material be made of thin laminations, the layers being alternatcly elastic and viscous. Assume that the layers are extremely thin, rela- 
tive to the wavelengths of the deformation field, so that the material behaves as a continuous medium of anisotropic properties. The initial stress is carried entirely by the elastic layers. We denote the elastic material by the index 1 and the viscous material by the index 2 . Hence $P_{2}=0$ and the average compressive stress in the composite medium is

$$
P=\alpha_{1} P_{1} \text {. }
$$

The moduli of the elastic layer are denoted by $M_{1}$ and $L_{1}$. Hence,

$$
\begin{aligned}
\bar{M}_{1} & =M_{1} \\
\bar{L}_{1} & =L_{1} .
\end{aligned}
$$

The properties of the viscous layers are assumed to be those of a Newtonian fluid of viscosity $\eta$ represented by the operators

$$
\bar{M}_{2}=\bar{L}_{2}=\eta p \text {. }
$$

Applying Eqs. 18, the operators for the composite medium are

Let us put

$$
\begin{aligned}
\bar{M} & =\alpha_{1} M_{1}+\alpha_{2} \eta p \\
\bar{L} & =\frac{1}{\frac{\alpha_{1}}{L_{1}}+\frac{\alpha_{2}}{\eta p}} .
\end{aligned}
$$

Hence,

$$
\begin{aligned}
\alpha_{1} M_{1} & =M \\
\alpha_{2} \eta & =M^{\prime} \\
L_{1} / \alpha_{1} & =L_{r} \\
\frac{\alpha_{2} L_{1}}{\alpha_{1} \eta} & =r .
\end{aligned}
$$

$$
\begin{aligned}
\bar{M} & =M+M^{\prime} p \\
\bar{L} & =\frac{p}{p+r} L_{r} .
\end{aligned}
$$

The operators $\bar{M}$ and $\bar{L}$ represent respectively a Kelvin type and Maxwell type model.

Write the characteristic Eq. 16 in the form

$$
\frac{1-\frac{4 \bar{M}}{P} \xi^{2}}{\left(1-\xi^{2}\right)^{2}}=\frac{\bar{L}}{P}
$$

After inserting expressions Eq. 25 for $\bar{M}$ and $\bar{L}$ and assuming $\xi$ is given, Eq. 26 determines the corresponding value of $p$. The properties of the roots $p$ are easily derived by plotting both sides of Eq. 26 as a function of $p$. The right hand side is a hyperbola with vertical and horizontal assymptotes represented by curve (a) in Fig. 1. The left hand side is a straight line (b) with a negative slope which always intersects the hyperbola at two points A and B. Hence the two roots for $p$ are real in conformity with a general theorem established 


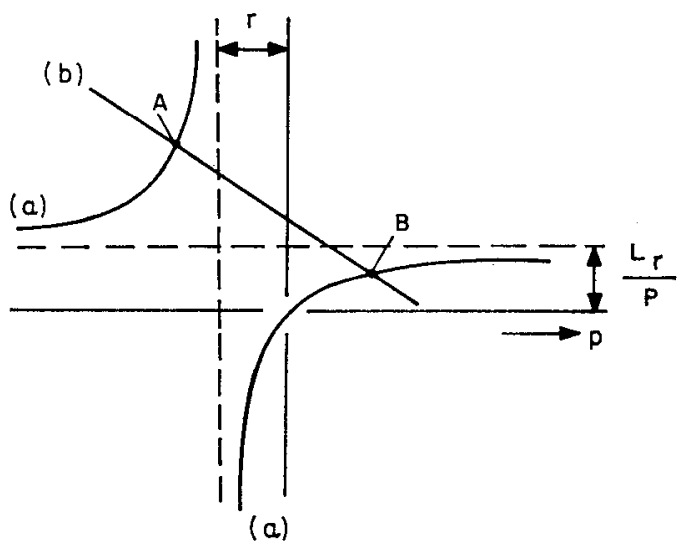

FIG. 1. Graphical construction for the roots $p$ of Eq. 26.

in $(2,5)$. Figure 1 shows that there is never more than one positive root. However, there may be no positive roots. The limiting case is obtained by putting $p=0$ into Eq. 26. This yields

$$
1-\frac{4 M}{P} \xi^{2}=0
$$

or

$$
\xi^{2}=\frac{P}{4 M}=\frac{P_{1}}{4 M_{1}}
$$

This result is interpreted as follows: as already shown in (1), $\xi$ determines a characteristic direction. The existence of characteristics is also illustrated by writing the solution Eq. 13 as a sum of trigonometric functions. For example, we write

$$
\cos l x \cos \frac{\pi y}{h}=\frac{1}{2} \cos l(x+\xi y)+\frac{1}{2} \cos l(x-\xi y)
$$

Hence Eq. 28 determines two limiting directions $\xi= \pm \frac{1}{2} \sqrt{P_{1} / M_{1}}$ of the characteristics in which the rate of growth of the deformation vanishes. In other words, the deformation can only propagate with characteristics whose angle in the normal direction of the layers is smaller than

$$
\theta=\tan ^{-1}\left[\frac{1}{2} \sqrt{P_{1} / M_{1}}\right]
$$

An initial disturbance will spread only inside a sector area limited by two characteristic directions with an inclination of angle $\theta$, as illustrated in Fig. 2 .

Examine the value of $p$ for a characteristic direction which is normal to the layers. This value is obtained by substituting $\xi=0$ in Eq. 26. The equation becomes

$$
P=\bar{l}
$$




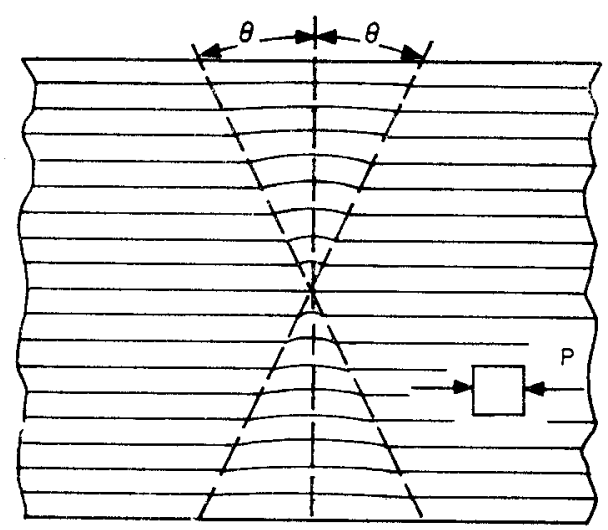

Fig. 2. Spreading of a disturbance due to internal instability of a viscoelastic anisotropic medium.

Solving this equation for $p$ yields

$$
p=\frac{r P}{L_{r}-P}
$$

This normal characteristic direction will generally correspond to the maximum value of $p$. In this case a disturbance will tend to propagatc in a direction normal to the layers.

It is theoretically possible for $p$ to be maximum in characteristic directions which are oriented symmetrically relative to the normal direction. This occurs when the left hand side of Eq. 26 increases with $\xi$ in the vicinity of $\xi=0$, i.e., if

$$
4 \bar{M} / P<2 .
$$

This inequality may be written

$$
2 M+2 M^{\prime} p<P .
$$

Since the elastic modulus $M$ is generally larger than $P$ the inequality Eq. 34 will be verified only in exceptional cases.

We have analyzed here a particular example illustrating internal instability of a viscoelastic medium. However, the qualitative features derived from this example will also be exhibited by viscoelastic media of a more general type. This is due to the property of expressions Eq. 10 for the operators $\bar{M}$ and $\bar{L}$ of being increasing functions of $p$. This property is a consequence of thermodynamic principles.

\section{Internal Instability of a Viscous Anisotropic Medium}

The general equations are rigorously applicable to a medium which is at rest when undisturbed under the initial stress, and purely viscous for incremental deformations superimposed in this initial state. Such a case may be considered to approximate certain types of creep and plastic behavior. The 
corresponding operators are

$$
\begin{aligned}
\bar{M} & =M^{\prime} p \\
\bar{L} & =L^{\prime} p .
\end{aligned}
$$

These represent a particular case of the general operators Eq. 10 in which only purely viscous terms are retained. The coefficient $M^{\prime}$ is a viscosity coefficient for an incremental tensile stress $t_{11}$ in the $x$ direction. In this case the stressstrain relation Eq. 8 becomes

$$
t_{11}=4 M^{\prime} \frac{d e_{x x}}{d t} .
$$

Similarly, the stress-strain relation Eq. 9 becomes

$$
\Delta_{x y}=2 L^{\prime} \frac{d e_{x y}}{d t} .
$$

Hence $L^{\prime}$ is a viscosity coefficient for a tangential stress parallel to the $x$ direction.

As previously stated, substitution of the operators into the characteristic Eq. 16 provides an exact solution for internal instability if the undisturbed medium is initially at rest.

IIowever, the results in this case are also applicable as an approximation to an anisotropic viscous fluid where the undisturbed initially stressed state is one of uniform flow, and the fluid is of such high viscosity that the inertia forces arc ncgligible. Therefore, we shall treat the problem in the context of the anisotropic fluid. The validity of this approximation will be discussed in the following section by comparison with an exact treatment for the case of a fluid.

An anisotropic fluid may also be considered as representing approximately the properties of a medium constituted by very thin alternating layers of viscous isotropic fluids of different viscosities. As established in preceding sections, this approximation is valid only for layers which are very thin in comparison with the wavelengths of the deformation field. The viscosities of the alternati $\eta \mathrm{g}$ layers are denoted by $\eta_{1}$ and $\eta_{2}$ and we assume that they occupy, respectively, fractions $\alpha_{1}$ and $\alpha_{2}$ of the total thickness. In this case operators for the two materials are

$$
\begin{aligned}
& \bar{L}_{1}=\bar{M}_{1}=\eta_{1} p \\
& \bar{L}_{2}=\bar{M}_{2}=\eta_{2} p .
\end{aligned}
$$

Applying Eqs. 18, the two viscosity coefficients of the composite material are derived by

$$
\begin{aligned}
M^{\prime} & =\alpha_{1} \eta_{1}+\alpha_{2} \eta_{2} \\
L^{\prime} & =\frac{1}{\frac{\alpha_{1}}{\eta_{1}}+\frac{\alpha_{2}}{\eta_{2}}} .
\end{aligned}
$$


This yields the coefficients in the operators Eq. 35. Substituting Eq. 35 into the characteristic Eq. 16, we obtain

$$
\frac{P}{L^{\prime} p}=\xi^{4}+2\left(\frac{2 M^{\prime}}{L^{\prime}}-1\right) \xi^{2}+1 .
$$

This is a relation between $p$ and $\xi$.

Before proceeding further consider carefully the physical significance of this result. Since we are discussing a viscous fluid under a compressive stress $P$ the medium is initially undergoing a deformation with uniform strain-ratc, scc Fig. 3. While being shortened in the $x$ direction it is expanding laterally by the same amount. At a certain instant $t=0$ the distance between the parallel boundaries is $h$. If the deformation remained uniform there would be a displacement field corresponding to this deformation but no incremental stress. The field Eq. 13 of the present theory represents the perturbation superposed upon the uniform strain-rate solution. The amplitude of this perturbation is proportional to the amplification factor $\exp (p t)$. According to relation Eq. 15 the value of $\xi$ in Eq. 40 defines the wavelength along $x$ of an initial perturbation. The amplitude of this perturbation will grow exponentially at a rate defied by the value of $p$ as determined by Eq. 40. In the numerical analysis it is convenient to introduce a nondimensional time scale. Consider an interval of time such that the overall shortening in the $x$ direction is about 10 per cent. The time $t_{1}$ required for this amount of shortening is determined by the equation

where we put

$$
P t_{1}=-4 M^{\prime} e_{x x}
$$

$$
e_{x x}=-0.1
$$

Hence

$$
t_{1}=0.4 \frac{M^{\prime}}{P}=0.4 R \frac{L^{\prime}}{P}
$$

where

$$
R=\frac{M^{\prime}}{L^{\prime}}
$$

is a measure of the viscous anisotropy. Using Eq. 40 and introducing the reference time $t_{1}$ we derive

with

$$
p t=Z \frac{t}{t_{1}}
$$

$$
\begin{aligned}
Z & =\frac{0.4 R z^{4}}{z^{4}+2(2 R-1) \pi^{2} z^{2}+\pi^{4}} \\
z & =l h=\frac{\pi}{\xi}=\frac{2 \pi h}{\mathscr{L}} .
\end{aligned}
$$

The amplification factor at time $t$ for any initial disturbance sinusoidal along $x$ is $\exp (p t)=\exp \left(Z t / t_{1}\right)$.

The exact theory is based on the assumption of a constant value of $h$ and $\mathcal{L}$. 
When applying the theory to a medium in a state of initial flow, $h$ and $\&$ will vary slightly during the deformation. To take this into account we may replace $\xi$ by an average value. As shown in the next section this amounts to using for $h$ the initial value and for $\mathscr{L}$ the final value.

Consider the case $R=10$. For small wavelengths the value of $z$ tends to infinity and $Z$ takes the limiting value $Z=4$. At the time $t=t_{1}$, i.e., for an overall compressive strain of about 10 per cent the amplification factor for small wavelengths is,

$$
\exp (Z)=\exp (4)=54.6
$$

which increases very rapidly for $t>t_{1}$.

For large wavelengths the value of $z$ tends to zero and the amplification factor tends to unity. Therefore the system acts as a high pass filter with a cutoff wavelength proportional to the distance $h$ between the two rigid boundaries. In addition this cut-off wavelength also depends on the anisotropy and tends to become smaller when $R=M^{\prime} / L^{\prime}$ is increased.

In order to bring out the filtering effect we shall evaluate the internal folding by considering a medium which is initially disturbed from perfect uniformity. The initial disturbance is assumed to be a vertical displacement field

$$
v=\frac{b}{1+\left(\frac{x}{a}\right)^{2}} \cos \frac{\pi y}{h} .
$$

This initial disturbance is illustrated schematically in Fig. 3. We introduce the Fourier integral representation

$$
v=b a \cos \frac{\pi y}{h} \int_{0}^{\infty} \exp (-l a) \cos l x d l .
$$

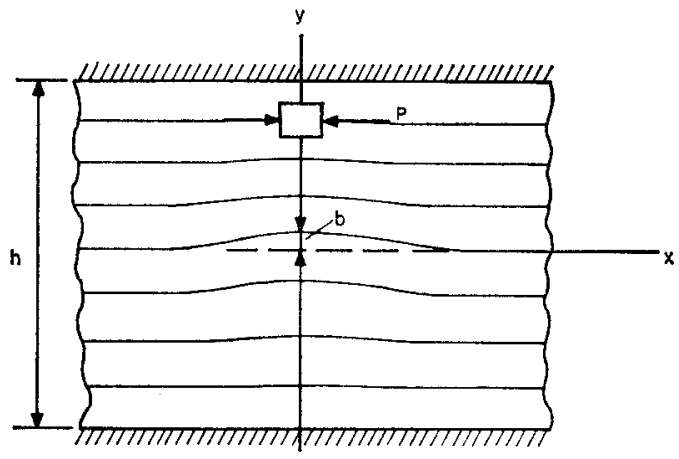

FIG. 3. Initial disturbance in viscous anisotropic medium under an initial compression $P$ between confining boundaries. 
As can be seen this expression represents a superposition of solutions of the type Eq. 13. After a time $t$ each elementary solution is multiplied by $\exp (p t)$. Hence, the time history of the displacement $v$ on the $x$ axis (for $y=0$ ) is given by

$$
v_{o}(x)=b a \int_{0}^{\infty} \exp (p t-l a) \cos l x d l .
$$

This integral may be written

$$
\frac{v_{0}(x)}{b}=\frac{a}{h} \int_{0}^{\infty} \exp \left(Z \frac{t}{t_{1}}-z \frac{a}{h}\right) \cos z \frac{x}{h} d z .
$$

It is a function of $x / h$ which contains three parameters $t / t_{1}, a / h$ and $R$. At the origin $(x=0)$ the displacement $v_{o}(0)$ is given by

$$
\frac{v_{o}(0)}{b}=\frac{a}{h} \int_{0}^{\infty} \exp \left(Z \frac{t}{t_{1}}-z \frac{a}{h}\right) d z
$$

Since $b$ is the maximum initial amplitude of the disturbance (at $x=0$ and $t=0)$ the ratio $v_{o}(0) / b$ represents the amplification at the time $t$.

Four numerical cases have been evaluated. These cases and the corresponding values of the amplification $v_{o}(0) / b$ are shown in Table I.

\section{TABLE I. Amplification factor $v_{o}(0) / b$ of the disturbance at $x=0$.}

$\begin{array}{lcrcc} & a / h & R & t / t_{1} & v_{0}(0) / b \\ \text { Case 1. } & 1 / 25 & 5 & 1.0 & 3.75 \\ \text { Case 2. } & 1 / 25 & 10 & 1.0 & 13.2 \\ \text { Case 3. } & 1 / 25 & 15 & 1.0 & 52.3 \\ \text { Case 4. } & 1 / 25 & 10 & 1.5 & 68.8\end{array}$

Significant amplification appears only for Cases 3 and 4 . This indicates that internal folding will not become appreciable unless the degree of anisotropy is sufficiently large. This requires the value of $R$ to be larger than about 10 to 15 .

The shape of the folding is given by Eq. 50 and is represented in Fig. 4 for the four cases listed in Table I. The initial disturbance represented by the bell-shaped curve is transformed into an oscillatory folding which is considerably sharper. This is to be expected since the system acts as a high-pass filter and tends to amplify the shorter wavelengths.

\section{Internal Instability of a Viscous Anisotropic Fluid Undergoing Finite Strain}

The previous analysis is rigorous when the medium is at rest in the state of initial stress. When this is not the case the result is still applicable as an approximation. In order to examine this point and establish the range of validity of the correspondence principle we shall derive a rigorous treatment for the case of an anisotropic fluid under initial stress. It is assumed that the fluid is incompressible and of sufficiently high viscosity that inertia forces may be neglected. 


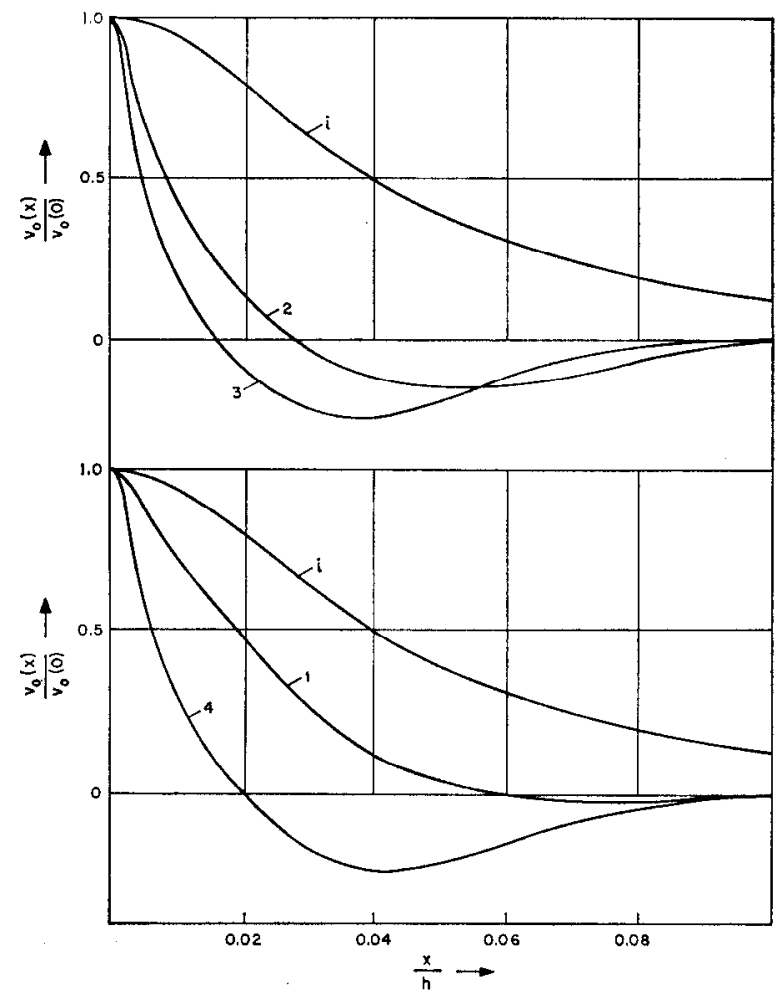

FIG. 4. Curve $i=$ shape of initial disturbance $(t=0)$ given by the equation $v / b$ $=1 /\left[1+(x / a)^{2}\right]$. Curves $1,2,3,4=$ shape of the folding for the corresponding cases of Table I.

As an example of fluid anisotropy consider again the case of a laminated medium constituted of very thin alternating layers of two different isotropic fluids of viscosities $\eta_{1}$ and $\eta_{2}$ occupying respectively fractions $\alpha_{1}$ and $\alpha_{2}$ of the total thickness. In directions parallel to the laminations the properties of the fluid are defined by two viscosity coefficients given by Eqs. 39,

$$
\begin{aligned}
& \eta_{n}=\alpha_{1} \eta_{1}+\alpha_{2} \eta_{2} \\
& \eta_{\iota}=\frac{1}{\frac{\alpha_{1}}{\eta_{1}}+\frac{\alpha_{2}}{\eta_{2}}} .
\end{aligned}
$$

As previously indicated such a layered material behaves as a continuous anisotropic medium only under the assumption that the layers are very thin compared to the wavelength of the deformation field.

Consider first a particular instant where the layers are oriented along the $x$ direction, and denote by $v_{x}$ and $v_{y}$ a two-dimensional velocity field at that instant. The stress is given by 
M. A. Biot

$$
\begin{aligned}
\sigma_{x x}-\sigma & =2 \eta_{n} \mathcal{E}_{x x}=2 \eta_{n} \frac{\partial v_{x}}{\partial x} \\
\sigma_{y y}-\sigma & =2 \eta_{n} \mathcal{E}_{y y}=2 \eta_{n} \frac{\partial v_{y}}{\partial y} \\
\sigma_{x y} & =2 \eta_{t} \mathcal{E}_{x y}=\eta_{t}\left(\frac{\partial v_{y}}{\partial x}+\frac{\partial v_{x x}}{\partial y}\right) .
\end{aligned}
$$

Incompressibility implies the additional equation

$$
\mathcal{E}_{x x}+\mathcal{E}_{y y}=0 .
$$

For constant principle stresses $S_{11}=\sigma_{x x}, S_{22}=\sigma_{y y},\left(\sigma_{x y}=0\right)$ the strain-rate is constant and uniform

$$
-\mathcal{E}_{x x}=\mathcal{E}_{y y}=\frac{S_{22}-S_{11}}{4 \eta_{n}}=\frac{P}{4 \eta_{n}}=p_{0} .
$$

The velocity field corresponding to this uniform strain rate is now perturbed by a small additional velocity field which we denote by the same notation $v_{x}$ and $v_{y}$. The perturbed strain-rate components are

$$
\begin{aligned}
\mathcal{E}_{x x} & =-p_{o}+\frac{\partial v_{x}}{\partial y} \\
\mathcal{E}_{y y} & =p_{o}+\frac{\partial v_{y}}{\partial y} \\
2 \mathcal{E}_{x y} & =\frac{\partial v_{y}}{\partial x}+\frac{\partial v_{x}}{\partial y} .
\end{aligned}
$$

The layers have also rotated through a small angle $\varphi$. Let $x^{\prime}, y^{\prime}$ be a coordinate system rotated through the same angle and denote by $\sigma_{x x}{ }^{\prime}, \sigma_{y y}{ }^{\prime}, \sigma_{x y}{ }^{\prime}, \mathcal{E}_{x x}{ }^{\prime}$, $\mathcal{E}_{y y}{ }^{\prime}, \mathcal{E}_{x y}{ }^{\prime}$ the stress and strain-rate components referred to these rotated axes see Fig. 5 . To the first order in $\varphi$ we write for the strain-rate components

$$
\begin{aligned}
& \mathcal{E}_{x x}{ }^{\prime}=\mathcal{E}_{x x} \\
& \mathcal{E}_{y y}{ }^{\prime}=\mathcal{E}_{y y} \\
& \mathcal{E}_{x y}{ }^{\prime}=\left(\mathcal{E}_{y y}-\mathcal{E}_{x x}\right) \varphi+\mathcal{E}_{x y}
\end{aligned}
$$

and for the stresses

$$
\begin{aligned}
& \sigma_{x x}=\sigma_{x x}{ }^{\prime} \\
& \sigma_{y y}=\sigma_{y y}{ }^{\prime} \\
& \sigma_{x y}=\left(\sigma_{x x}{ }^{\prime}-\sigma_{y y}{ }^{\prime}\right) \varphi+\sigma_{x y}{ }^{\prime} .
\end{aligned}
$$

On the other hand Eqs. 54 remain valid for the rotated axes, hence

$$
\begin{aligned}
\sigma_{x x}{ }^{\prime}-\sigma & =2 \eta_{n} \mathcal{E}_{x x}{ }^{\prime} \\
\sigma_{y y}{ }^{\prime}-\sigma & =2 \eta_{n} \mathcal{E}_{y y}{ }^{\prime} \\
\sigma_{x y}{ }^{\prime} & =2 \eta_{t} \mathcal{E}_{x y}{ }^{\prime} .
\end{aligned}
$$




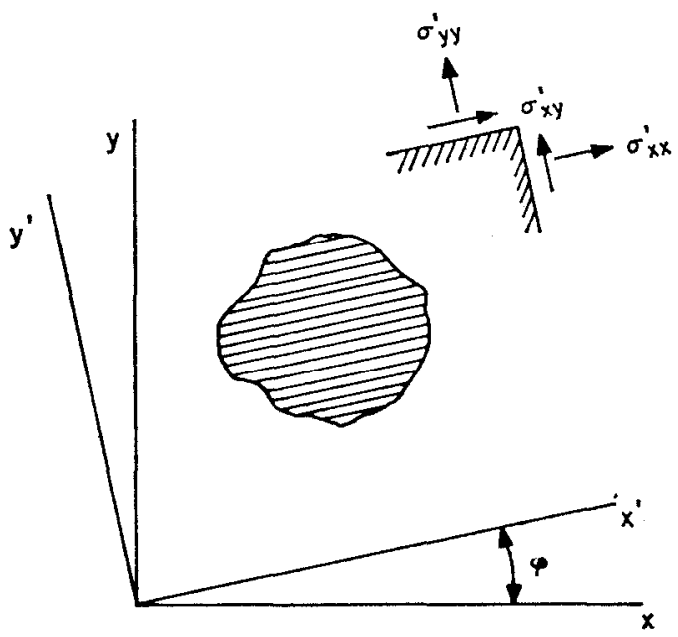

FIG. 5. Stresses in an anisotropic fluid referred to rotated axes.

From Eqs. 58, 59 and 60 we derive

$$
\begin{aligned}
\sigma_{x x}-\sigma & =2 \eta_{n} \mathcal{E}_{x x} \\
\sigma_{y y}-\sigma & =2 \eta_{n} \mathscr{E}_{y y} \\
\sigma_{x y} & =2\left(\eta_{n}-\eta_{t}\right)\left(\mathcal{E}_{x x}-\mathcal{E}_{y y}\right) \varphi+2 \eta_{t} \mathcal{E}_{x y} .
\end{aligned}
$$

Note that in the last equation by using the values Eqs. 57 we may write approximately to the same order,

$$
\left(\mathcal{E}_{x x}-\mathcal{E}_{y y}\right) \varphi=-2 p_{\circ} \varphi .
$$

Equations 61 relate the stress to the strain-rate in terms of components referred to fixed axes $x$ and $y$.

In addition we must satisfy the condition of equilibrium of the stress field namely

$$
\begin{aligned}
& \frac{\partial \sigma_{x x}}{\partial x}+\frac{\partial \sigma_{x y}}{\partial y}=0 \\
& \frac{\partial \sigma_{x y}}{\partial x}+\frac{\partial \sigma_{y y}}{\sigma y}=0
\end{aligned}
$$

while the condition of incompressibility Eq. 55 is verified by putting

$$
\begin{aligned}
& v_{x}=\frac{\partial \psi}{\partial y} \\
& v_{y}=-\frac{\partial \psi}{\partial x} .
\end{aligned}
$$

It is easy to eliminate all variables except $\psi$ and $\varphi$ between Eqs. 61, 63 and 64 . 


\section{A. Biot}

Keeping only first order terms we find

$\eta_{t} \frac{\partial^{4} \psi}{\partial x^{4}}+2\left(2 \eta_{n}-\eta_{i}\right) \frac{\partial^{4} \psi}{\partial x^{2} \partial y^{2}}+\eta_{t} \frac{\partial^{4} \psi}{\partial y^{4}}+4\left(\eta_{n}-\eta_{t}\right) p_{o}\left(\frac{\partial^{2} \varphi}{\partial x^{2}}-\frac{\partial^{2} \varphi}{\partial y^{2}}\right)=0$.

This equation may be expressed in terms of $v_{y}$ by taking its partial derivative with respect to $x$ and taking into account Eqs. 64, we obtain

$$
\begin{aligned}
\eta_{t} \frac{\partial^{4} v_{y}}{\partial x^{4}}+2\left(2 \eta_{n}-\eta_{t}\right) \frac{\partial^{4} v_{y}}{\partial x^{2} \partial y^{2}}+\eta_{t} \frac{\partial^{4} v_{y}}{\partial y^{4}} & \\
& +4\left(\eta_{n}-\eta_{t}\right) p_{0}\left(\frac{\partial^{3} \varphi}{\partial x \partial y^{2}}-\frac{\partial^{3} \varphi}{\partial x^{3}}\right)=0 .
\end{aligned}
$$

Note again that this equation governs the distribution of the velocity component $v_{y}$ and of the slope $\varphi$ at any particular instant as functions of fixed coordinates $x, y$. Consider now the unperturbed finite flow of the fluid under the stresses $S_{11}, S_{22}$. With a suitable choice of origin a fluid particle originally of coordinates $X, Y$ at $t=0$ will acquire at time $t$ the coordinates

$$
\begin{aligned}
& x=X e^{-p_{0} t} \\
& y=Y e^{p_{0} t} .
\end{aligned}
$$

When the system is perturbed, the $y$ coordinate of a particle may be represented as

$$
y_{1}=[Y+v(X, Y, t)] e^{p_{0} t}
$$

its $x$ coordinate is denoted by $x_{1}$. The component $v_{y}(t, x, y)$ of the perturbation velocity at the point $x_{1} y_{1}$ and the time $t$ is

$$
v_{y}\left(t, x_{1}, y_{1}\right)=\dot{y}_{1}-p_{o} y_{1}=\dot{v} e^{p_{o} t} .
$$

The dot denotes the partial derivative with respect to the time for $X$ and $Y$ constant. To the first order the perturbation velocity at the point $x_{1} y_{1}$ is the same as the perturbation velocity $v_{y}(t, x, y)$ at the point $x y$. Hence

$$
v_{y}(t, x, y)=\dot{v} e^{p_{o t}} \text {. }
$$

Also to the first order the slope $\varphi$ at the point $x y$ is

$$
\varphi=\frac{\partial v}{\partial x} e^{p_{o} t}
$$

Substitution of Eqs. 70 and 71 into Eq. 66 yields

$$
\begin{aligned}
\eta_{t} \frac{\partial^{4} \dot{v}}{\partial x^{4}}+2\left(2 \eta_{n}-\eta_{t}\right) \frac{\partial^{4} \dot{v}}{\partial x^{2} \partial y^{2}}+\eta_{t} \frac{\partial^{4} \dot{v}}{\partial y^{4}} & \\
& +4 p_{o}\left(\eta_{n}-\eta_{t}\right)\left(\frac{\partial^{4} v}{\partial x^{2} \partial y^{2}}-\frac{\partial^{4} v}{\partial x^{4}}\right)=0 .
\end{aligned}
$$


Assume a sinusoidal distribution of incremental displacement analogous to expression Eq. 13, namely

$$
v=\mathfrak{v} \cos l_{o} X \cos \pi \frac{Y}{h_{o}}=\mathfrak{v} \cos l x \cos \frac{\pi y}{h} .
$$

This corresponds to a confined medium illustrated in Fig. 3 with $l=l_{o} \exp \left(p_{o} t\right)$ and $h=h_{o} \exp \left(p_{o} t\right)$. We derive

$$
\dot{v}=\dot{\mathfrak{v}} \cos l x \cos \frac{\pi y}{h} .
$$

Substitution of Eqs. 73 and 74 into Eq. 72 yields

$$
\frac{\eta_{t} p}{P}=\left(1-\frac{\eta_{t}}{\eta_{n}}\right) \frac{1-\xi^{2}}{1+2\left(2 \frac{\eta_{n}}{\eta_{t}}-1\right) \xi^{2}+\xi^{4}}
$$

with

$$
p=\frac{\dot{\mathfrak{v}}}{\mathfrak{v}} \quad \text { and } \quad \xi=\frac{\pi}{l h}=\frac{\pi}{l_{o} h_{o}} e^{-2 p_{o} t}
$$

Note that $\xi$ is a function of time, hence also $p$. Therefore, a simple quadrature yields the time history of the small perturbation $\mathfrak{b}$ while the medium undergoes any amount of finite compresive strain. Actually when the compressive strain is not too large, say 10 per cent, the value of $\xi$ may be replaced by a constant average value

$$
\xi_{\text {av }} \cong \frac{\pi}{l_{o} h_{o}} e^{-p_{o} t}=\frac{\pi}{l h_{o}}
$$

Hence we may use the initial value $h_{o}$ of $h$ and the final wavelength $\mathfrak{L}=2 \pi / l$ at time $t$ in order to describe the result in terms of constant parameters.

We put $L^{\prime}=\eta_{t}$ and $M^{\prime}=\eta_{r}$ and assume that the anisotropy is large enough for significant instability to occur, hence we assume $\eta_{n} / \eta_{t}>10$. In that case the values of $p$ given by Eqs. 40 and 75 are approximately the same. The factor $1-\left(\eta_{t} / \eta_{n}\right)$ corresponds to a slight correction in the time scale. This verifies our assumption that the numerical solution of section 4 is valid as an approximation for a viscous fluid if the instability is significant.

It is interesting to note that according to Eq. 75 the perturbation is stable for $\xi>1$, i.e., for characteristic directions which lie at an angle less than $45^{\circ}$ with the $x$ direction. It also means that the large wavelengths are stable. This behavior is not shown by Eq. 40. However, the difference occurs only in the range of small values of $p$ where it does not appreciably affect the result.

Note that for an isotropic fluid $\left(\eta_{n}=\eta_{t}\right)$ the instability vanishes, as should be. Equation 75 is also valid in the complete range of the ratio $\eta_{n} / \eta_{t}$ and may be discussed in analogy with the elastic problem as developed in $(1,2)$. 
M. A. Biot

\section{References}

(1) M. A. Biot, "Internal Buckling under Initial Stress in Finite Elasticity," Proc. Royal Society, A, Vol. 273, pp. 306-328, 1963.

(2) M. A. Biot, "Mechanics of Incremental Deformations," New York, John Wiley \& Sons, Inc., 1965.

(3) M. A. Biot, "Theory of Internal Buckling of a Confined Multilayered Structure," Geological Society of America Bulletin, Vol. 75, No. 6, pp. 563-568, 1964.

(4) M. A. Biot, "On the Instability and Folding Deformation of a Layered Viscoelastic Medium in Compression," Jour. Appl. Mech., Ser. E, Vol. 26, pp. 393-400, 1959.

(5) M. A. Biot, "Stability of Multilayered Continua Including the Effect of Gravity and Viscoelasticity," Jour. Frank. Inst., Vol. 276, No. 3, pp. 231-252, 1963. 